Original article

Received: 10 April 2017 / Accepted: 20 September 2017

\title{
ACCURACY ASSESSMENT IN DETERMINING THE LOCATION OF CORNERS OF BUILDING STRUCTURES USING A COMBINATION OF VARIOUS MEASUREMENT METHODS
}

\author{
Robert Krzyżek, Anna Przewięźlikowska
}

AGH University of Science and Technology, Faculty of Mining Surveying and Environmental Engineering, Department of Integrated Geodesy and Cartography

\begin{abstract}
When surveys of corners of building structures are carried out, surveyors frequently use a compilation of two surveying methods. The first one involves the determination of several corners with reference to a geodetic control using classical methods of surveying field details. The second method relates to the remaining corner points of a structure, which are determined in sequence from distance-distance intersection, using control linear values of the wall faces of the building, the so-called tie distances. This paper assesses the accuracy of coordinates of corner points of a building structure, determined using the method of distance-distance intersection, based on the corners which had previously been determined by the conducted surveys tied to a geodetic control. It should be noted, however, that such a method of surveying the corners of building structures from linear measures is based on the details of the first-order accuracy, while the regulations explicitly allow such measurement only for the details of the second- and third-order accuracy. Therefore, a question arises whether this legal provision is unfounded, or whether surveyors are acting not only against the applicable standards but also without due diligence while performing surveys? This study provides answers to the formulated problem. The main purpose of the study was to verify whether the actual method which is used in practice for surveying building structures allows to obtain the required accuracy of coordinates of the points being determined, or whether it should be strictly forbidden. The results of the conducted studies clearly demonstrate that the problem is definitely more complex. Eventually, however, it might be assumed that assessment of the accuracy in determining a location of corners of a building using a combination of two different surveying methods will meet the requirements of the regulation [MIA, 2011), subject to compliance with relevant baseline criteria, which have been presented in this study. Observance of the proposed boundary conditions would allow for frequent performance of surveys of building structures by surveyors (from tie distances), while maintaining the applicable accuracy criteria. This would allow for the inclusion of surveying documentation into the national geodetic and cartographic documentation center database pursuant to the legal bases.
\end{abstract}

Keywords: building structure, surveys, distance-distance intersection, analysis of accuracy 


\section{Introduction}

The principles for carrying out horizontal surveys of field details constitute elementary knowledge necessary for the performance of geodetic surveys. Both this knowledge and the binding legal regulations in Poland (MIA, 2011), §29 section 1 "A geodetic horizontal survey is performed as to ensure that the position of a point, in relation to the nearest points of the horizontal geodetic control and of the measurement control, is determined with an accuracy of not less than $0.10 \mathrm{~m}$ in the case of field details of the first-order accuracy..." state unambiguously that field details of the first-order accuracy can only be measured referring to the geodetic control. Apparently, it is very easy in interpretation as well as in practical application. However, the implementation of such tasks is not quite so clear.

The complexity of buildings and structures has increased significantly over the past 20-30 years which, to great extent, is influenced by high density of development. Therefore, special surveying monitoring services are being established to capture realtime information on its current state. In order to create information data sets, specialists have to carry out accurate measurements, which are often difficult due to the large density of new buildings and quaint shapes of engineering structures (Andrianova, 2008), (Buśko 2016a).

When performing surveys of the corners of building structures, surveyors frequently use a compilation of two surveying methods. The first stage involves the determination of several corners to a geodetic control using classical methods of surveying field details, under (MIA, 2011), § 32 "Geodetic horizontal surveys are performed using the following methods: precision positioning with GNSS, polar method, orthogonal method (rectangular offset sampling) as well as: angular intersection, distance-distance intersection, angular-distance intersection". If there is no possibility of a direct survey of a corner to the geodetic control, in the second stage surveyors use distance-distance intersection as an indirect method, based on two base points.

The method of distance-distance intersection is most frequently used to determine the remaining corner points of a structure, using control linear measures of the wall faces of the building, the so-called tie distances. This paper assesses the accuracy of coordinates of corner points of a building structure, determined using the method of distance-distance intersection, based on the corners which had previously been determined by the conducted surveys tied to a geodetic control. Such surveying practice is also often accepted when basic trig data are incorporated into the national geodetic and cartographic documentation center databases. It should be noted, however, that the described method of surveying corners of building structures from linear measures (tie distances) is based on the details of the first-order accuracy rather than on the geodetic control. This is inconsistent with (MIA, 2011), § 32 section 4 "When measuring field details of the second- and third-order accuracy, in the absence of the points referred to in section 3, the position of the instrument and the reference points may be field details of the first-order accuracy". Therefore, a question arises whether this legal provision is unfounded, or whether surveyors are acting not only against the applicable standards but also without due diligence during the performance of surveys? This study provides answers to the formulated problem.

The main purpose of the research study was to verify whether the actual method which is used in practice for the implementation of the surveys of building structures allows to obtain the required accuracy of the coordinates of the points being determined, or whether it should be strictly forbidden. The results of the conducted studies clearly demonstrate that the problem is definitely more complex. 
It should also be mentioned that the methods presented in this paper may not be used in the process of setting-out building structures. This reservation is due to the accuracy requirements of setting out such objects.

At the same time, before the commencement of the surveys, the following questions should be answered: why the building is to be surveyed, which elements of the building shall be subjected to surveys, and what technology is to be used for the surveys (Ostwald \& Vaughan, 2016).

Frequently, the errors resulting from the problems in identifying elements of a building structure subjected to the performed surveys influence the results of surveys of the corners more than the surveying technology itself, as discussed in (Przewięźlikowska \& Krzyżek, 2016) and (Buśko, 2016b).

\section{Description of the performed studies}

The theoretical test structure of the performed research analysis was a building consisting of several corner points (Fig. 1). Points $A-E$ were measured with reference to the geodetic control, for which the rectangular coordinates $\mathrm{X}, \mathrm{Y}$ were obtained with the point position error $m_{P}$. The issues related to the position error $m_{P}$ of the points $A$ $E$ will be discussed later in this paper. At this point, it should be emphasized that the method used for the measurements of the corners $A-E$ of the building must meet the requirements of the (MIA, 2011) regulation, and most frequently it is tacheometry or RTN GNSS technology. The following step is the control survey of the wall face of the building $l_{i}$ (the so-called tie distance) with a linear measurement, e.g. an open-frame tape measure, with a mean error $m_{l_{i}}$.

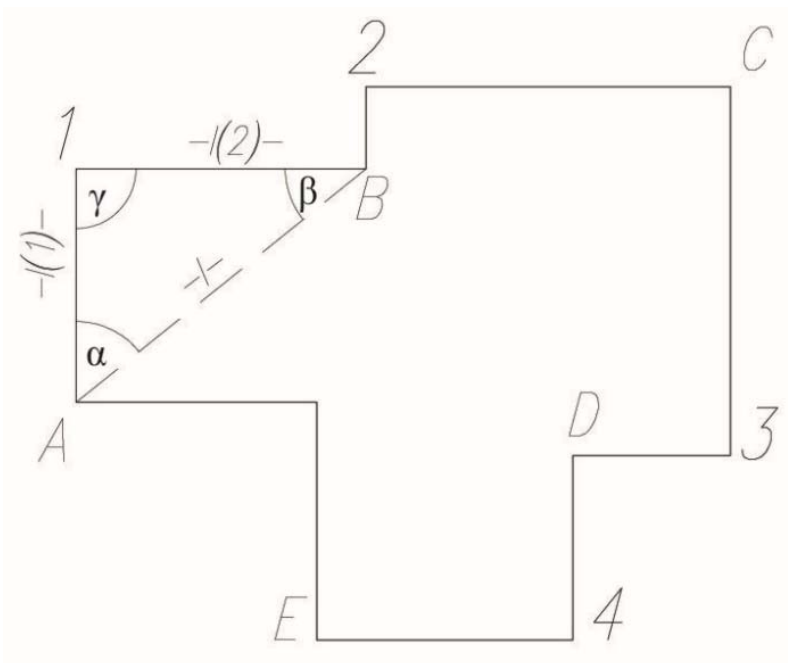

Fig. 1. Studied building structure

Basing on the $\mathrm{X}, \mathrm{Y}$ coordinates of the points $A-E$ and the measured tie distances $l_{i}$, the rectangular coordinates $\mathrm{X}, \mathrm{Y}$ of the remaining corner points 1-4 of the building are calculated using the classical method of distance-distance intersection. For the analyzed structure (Fig. 1), the denotations which were used in the formulas (1-6), referred only to one selected corner - No.1. The formula (1) illustrates the calculation of the coordinates of the corner point, basing solely on one of the base points $A, B$. 


$$
\begin{gathered}
X_{1}=X_{A}+l_{1} \cdot \cos A_{A 1} \\
Y_{1}=Y_{A}+l_{1} \cdot \sin A_{A 1}
\end{gathered}
$$

Having determined the $X, Y$ coordinates of the corner point No. 1, propagation of error is applied to a given function to obtain the formula (2) for the mean component errors of the coordinates $m_{X_{1}}, m_{Y_{1}}$, of the corner point No. 1 .

$$
\begin{gathered}
m_{X_{1}}= \pm \sqrt{\begin{array}{r}
m_{X_{A}}^{2}+\left[\cos \left(A_{A B}-\alpha\right)\right]^{2} \cdot m_{l_{1}}^{2}+\left[l_{1} \cdot\left(-\sin \left(A_{A B}-\alpha\right)\right)\right]^{2} \cdot m_{A_{A B}}^{2}+ \\
+\left[l_{1} \cdot \sin \left(A_{A B}-\alpha\right)\right]^{2} \cdot m_{\alpha}^{2}
\end{array}} \\
m_{Y_{1}}= \pm \sqrt{\begin{array}{c}
m_{Y_{A}}^{2}+\left[\sin \left(A_{A B}-\alpha\right)\right]^{2} \cdot m_{l_{1}}^{2}+\left[l_{1} \cdot \cos \left(A_{A B}-\alpha\right)\right]^{2} \cdot m_{A_{A B}}^{2}+ \\
+\left[l_{1} \cdot \cos \left(A_{A B}-\alpha\right)\right]^{2} \cdot m_{\alpha}^{2}
\end{array}}
\end{gathered}
$$

In the second stage, mean position error $M_{P_{1}}$ of the corner No.1 is calculated formula (3):

$$
M_{P_{1}}= \pm \sqrt{m_{X_{1}}^{2}+m_{Y_{1}}^{2}}= \pm \sqrt{m_{X_{A}}^{2}+m_{Y_{A}}^{2}+m_{l_{1}}^{2}+l_{1}^{2} \cdot\left(m_{A_{A B}}^{2}+m_{\alpha}^{2}\right)}
$$

where:

$m_{X_{A}}, m_{Y_{A}}-$ mean component errors of the position coordinates $\mathrm{X}, \mathrm{Y}$ of the point $\mathrm{A}$, $m_{l_{1}}$ - mean error of the linear measurement $l_{1}$ (tie distance) of the wall face of the building,

$l_{1}$ - length of the measured wall face of the building,

$m_{A_{A B}}$ - mean azimuth error of the side $A B$ calculated from the coordinates,

$m_{\alpha^{-}}$mean error of the horizontal angle $\alpha$ in the triangle 1-A-B calculated from

Carnot's theorem.

It is obvious that the mean component errors of the coordinates $X, Y$ of the base points $A$ and $B$ will be different. Nevertheless, to simplify the calculation and also to increase the accuracy requirements, we can assume that $m_{X_{A}}=m_{Y_{A}}=m_{X_{B}}=m_{Y_{B}}=$ $m_{P}$. The term "increased accuracy requirements" is to be interpreted in the way that one of the mean component errors of the coordinate (e.g. $m_{X_{A}}$ ) of a lower value can be artificially equalized with the second mean component error of the coordinate of a greater value (e.g. $m_{Y_{A}}$ ) which, in turn, increases the criterion of the requirements that should met by the final calculation results for the mean position error of the point $M_{P_{1}}$. Taking into account the above assumption, i.e. $m_{X_{A}}=m_{Y_{A}}=m_{X_{B}}=m_{Y_{B}}=m_{P}$, it is possible to calculate the mean azimuth error $m_{A_{A B}}$ of the side $A B$ calculated from the coordinates (formula 4):

$$
m_{A_{A B}}= \pm \frac{m_{P}}{l} \cdot \sqrt{1+2 \cdot \Delta y_{A B}^{2}}
$$

where:

$l$ - length of the side of the $A B$ base calculated from the coordinates. 
Assuming also that the mean linear measurement error for each wall face of the building is the same, i.e. $m_{l_{1}}=m_{l_{2}}=m_{l l}$, we can calculate the mean error $m_{\alpha}$ of the horizontal angle $\alpha$ in the triangle $1-A-B$ calculated from the coordinates (formula 5 ).

where:

$$
m_{\alpha}= \pm \frac{l_{2}}{l \cdot l_{1} \cdot \sqrt{1-\left(\frac{l^{2} \cdot l_{1}^{2}-l_{2}^{2}}{2 \cdot l \cdot l_{1}}\right)^{2}}} \cdot \sqrt{\cos ^{2} \beta \cdot m_{l}^{2}+(\cos \gamma-1)^{2} \cdot m_{l^{\prime}}^{2}}
$$

$$
m_{l}= \pm \sqrt{2} \cdot m_{P}
$$

\section{Processing and analysis of the research results}

In order to be provided with an answer to the formulated research thesis, three computational variants were performed with reference to the formula (2). In each of the variants presented, the mean position errors of the corners measured with reference to the control $\left(m_{P}\right)$ were the output variables. The mean errors $m_{P}$ were adopted at the level of $\pm 0.01 \mathrm{~m}$ for variant $1, \pm 0.02 \mathrm{~m}$ for variant $2, \pm 0.03 \mathrm{~m}$ for variant 3 . Such an assumption stems from two reasons. First of all, a practical approach was used, and therefore, such values of the errors $m_{P}$ were predetermined which are most frequently identified in topographic surveys of the details of the first-order accuracy (although $\left.m_{P_{\max }}= \pm 0.10 \mathrm{~m}\right)$. Secondly, as it will be demonstrated by the research results, the values higher than $\pm 0.03 \mathrm{~m}$ negatively affect the final results and virtually disqualify the surveying mode which is used by surveyors, presented in the research thesis. For each of the variants, the mean errors of the linear measurement $l_{i}$ (tie distance) of the wall face of the building $\left(m_{l_{1}}\right)$ were predetermined at the same value of $\pm 0.01 \mathrm{~m}$. The substantiation of this assumption results from the fact that adoption of higher values of $m_{l_{1}}$ (e.g. $\pm 0.02 \mathrm{~m}$ or $\pm 0.03 \mathrm{~m}$ ) does not affect the final research results and the value of $m_{l_{1}}= \pm 0.01 \mathrm{~m}$ is the standard value for such measurements.

The results of the conducted research experiment are presented as a formation of a mean position error of the corner of the building $M_{P}$ (formula 3 ) for each of the variants 1-3, both in the tabular form (Tables 1-3) and as a common plot (Fig. 2):

The plot illustrated in Fig. 2 is a result of compression of the data contained in Tables 1-3. It demonstrates the values exceeding the permissible mean error of point position $M_{P}$, depending on the lengths of the measured tie distances and the analyzed variants of the errors $m_{P}$. When analyzing the results presented in Tables 1-3 and in Fig. 2, the permissible mean position error of the corner of the building, i.e. $M_{P}= \pm 0.10 \mathrm{~m}$ was adopted as a reference point (MIA, 2011), § 29 section 1.

Tab. 1 and Fig. 1 clearly demonstrate that for the predetermined assumptions in variant 1 , the accuracy criterion $M_{P}= \pm 0.10 \mathrm{~m}$ is satisfied by those corners of the building for which one of the linear dimensions (tie distance) did not exceed $7.00 \mathrm{~m}$ from the corners of the building determined with reference to the control. Therefore, it can be assumed that for some corners of the building it would be acceptable to apply the subject measurement mode. Such situations often occur during surveys of buildings with wall lengths which are slightly shorter than typical, e.g. single or double garages, where these values are often at the levels of $5 \mathrm{~m}, 6 \mathrm{~m}$ or $7 \mathrm{~m}$. Generally, however, surveyors use a combination of measurement methods when surveying vestibules, outbuildings, stairs, etc., where the linear values of the walls often fall within 2-4 meters, producing the mean position error of the corner $M_{P}$ at the level of $\pm 0.05 \mathrm{~m}$ to $\pm 0.06 \mathrm{~m}$. 
Table 1. Mean position error of the corner of the building $M_{P}$ for variant 1 , where $\mathrm{mp}= \pm 0.01 \mathrm{~m}$

\begin{tabular}{|c|c|c|c|c|c|c|c|c|c|c|c|c|}
\hline & \multicolumn{12}{|c|}{$\mathrm{Mp}[\mathrm{m}]$} \\
\hline L1 [m] & 1.00 & 2.00 & 3.00 & 4.00 & 5.00 & 6.00 & 7.00 & 8.00 & 9.00 & 10.00 & 12.50 & 15.00 \\
\hline 1.00 & 0.023 & 0.024 & 0.024 & 0.024 & 0.024 & 0.024 & 0.024 & 0.024 & 0.024 & 0.024 & 0.024 & 0.024 \\
\hline 2.00 & 0.025 & 0.029 & 0.031 & 0.033 & 0.033 & 0.034 & 0.034 & 0.034 & 0.034 & 0.034 & 0.034 & 0.034 \\
\hline 3.00 & 0.026 & 0.032 & 0.037 & 0.040 & 0.042 & 0.043 & 0.044 & 0.045 & 0.045 & 0.045 & 0.046 & 0.046 \\
\hline 4.00 & 0.026 & 0.033 & 0.040 & 0.045 & 0.049 & 0.051 & 0.053 & 0.055 & 0.056 & 0.056 & 0.058 & 0.058 \\
\hline 5.00 & 0.026 & 0.034 & 0.042 & 0.049 & 0.054 & 0.058 & 0.061 & 0.063 & 0.065 & 0.066 & 0.069 & 0.070 \\
\hline 6.00 & 0.026 & 0.035 & 0.044 & 0.052 & 0.058 & 0.064 & 0.068 & 0.071 & 0.074 & 0.076 & 0.079 & 0.081 \\
\hline 7.00 & 0.026 & 0.035 & 0.045 & 0.054 & 0.061 & 0.068 & 0.073 & 0.077 & 0.081 & 0.084 & 0.089 & 0.092 \\
\hline 8.00 & 0.026 & 0.035 & 0.045 & 0.055 & 0.064 & 0.071 & 0.078 & 0.083 & 0.087 & 0.091 & 0.098 & 0.102 \\
\hline 9.00 & 0.026 & 0.035 & 0.046 & 0.056 & 0.066 & 0.074 & 0.081 & 0.087 & 0.092 & 0.097 & 0.105 & \\
\hline 10.00 & 0.026 & 0.036 & 0.046 & 0.057 & 0.067 & 0.076 & 0.084 & 0.091 & 0.097 & 0.102 & & \\
\hline 12.50 & 0.026 & 0.036 & 0.047 & 0.058 & 0.069 & 0.080 & 0.089 & 0.098 & 0.106 & & & \\
\hline 15.00 & 0.026 & 0.036 & 0.047 & 0.059 & 0.071 & 0.082 & 0.092 & 0.102 & & & & \\
\hline
\end{tabular}

Table 2. Mean position error of the corner of the building $M_{P}$ for variant 2, where $\mathrm{mp}= \pm 0.02 \mathrm{~m}$

\begin{tabular}{|c|c|c|c|c|c|c|c|c|c|c|c|c|}
\hline & \multicolumn{12}{|c|}{$\mathrm{Mp}[\mathrm{m}]$} \\
\hline$\underset{\mathrm{L} 1[\mathrm{~m}]}{\mathrm{L2}]}$ & 1.00 & 2.00 & 3.00 & 4.00 & 5.00 & 6.00 & 7.00 & 8.00 & 9.00 & 10.00 & 12.50 & 15.00 \\
\hline 1.00 & 0.040 & 0.041 & 0.042 & 0.042 & 0.042 & 0.042 & \begin{tabular}{|l|}
0.042 \\
\end{tabular} & 0.042 & 0.042 & 0.042 & 0.042 & 0.042 \\
\hline 2.00 & 0.044 & 0.053 & 0.058 & 0.060 & 0.062 & 0.063 & 0.063 & 0.064 & 0.064 & 0.064 & 0.064 & 0.064 \\
\hline 3.00 & 0.046 & 0.059 & 0.069 & 0.076 & 0.080 & 0.083 & \begin{tabular}{|l|}
0.085 \\
\end{tabular} & 0.086 & 0.087 & 0.087 & 0.088 & 0.089 \\
\hline 4.00 & 0.046 & 0.062 & 0.077 & 0.087 & 0.095 & 0.100 & & & & & & \\
\hline 5.00 & 0.046 & 0.064 & 0.081 & 0.095 & 0.106 & & & & & & & \\
\hline 6.00 & 0.047 & 0.065 & 0.084 & 0.101 & & & & & & & & \\
\hline 7.00 & 0.047 & 0.066 & 0.086 & 0.105 & & & & & & & & \\
\hline 8.00 & 0.047 & 0.066 & 0.088 & 0.108 & & & & & & & & \\
\hline 9.00 & 0.047 & 0.067 & 0.089 & 0.110 & & & & & & & & \\
\hline 10.00 & 0.047 & 0.067 & 0.089 & 0.111 & & & & & & & & \\
\hline 12.50 & 0.047 & \begin{tabular}{|l|}
0.067 \\
\end{tabular} & 0.090 & 0.114 & & & & & & & & \\
\hline 15.00 & 0.047 & 0.067 & 0.091 & 0.115 & & & & & & & & \\
\hline
\end{tabular}


Table 3. Mean position error of the corner of the building $M_{P}$ for variant 3, where $\mathrm{mp}= \pm 0.03 \mathrm{~m}$

\begin{tabular}{|c|c|c|c|c|c|c|c|c|c|c|c|c|}
\hline & \multicolumn{12}{|c|}{$\mathrm{Mp}[\mathrm{m}]$} \\
\hline $\mathrm{L1}[\mathrm{m}]$ & 1.00 & 2.00 & 3.00 & 4.00 & 5.00 & 6.00 & 7.00 & 8.00 & 9.00 & 10.00 & 12.50 & 15.00 \\
\hline 1.00 & 0.058 & 0.060 & 0.061 & 0.061 & 0.061 & 0.061 & 0.062 & 0.062 & 0.062 & 0.062 & 0.062 & 0.062 \\
\hline 2.00 & 0.064 & 0.078 & 0.085 & 0.089 & 0.091 & 0.093 & 0.093 & 0.094 & 0.094 & 0.095 & 0.095 & 0.095 \\
\hline 3.00 & 0.067 & 0.087 & 0.103 & & & & & & & & & \\
\hline 4.00 & 0.067 & 0.092 & 0.114 & & & & & & & & & \\
\hline 5.00 & 0.068 & 0.095 & 0.121 & & & & & & & & & \\
\hline 6.00 & 0.068 & 0.096 & 0.125 & & & & & & & & & \\
\hline 7.00 & 0.068 & 0.097 & 0.128 & & & & & & & & & \\
\hline 8.00 & 0.068 & 0.098 & 0.130 & & & & & & & & & \\
\hline 9.00 & 0.068 & 0.099 & 0.132 & & & & & & & & & \\
\hline 10.00 & 0.068 & 0.099 & 0.133 & & & & & & & & & \\
\hline 12.50 & 0.068 & 0.099 & 0.135 & & & & & & & & & \\
\hline 15.00 & 0.068 & 0.100 & & & & & & & & & & \\
\hline
\end{tabular}

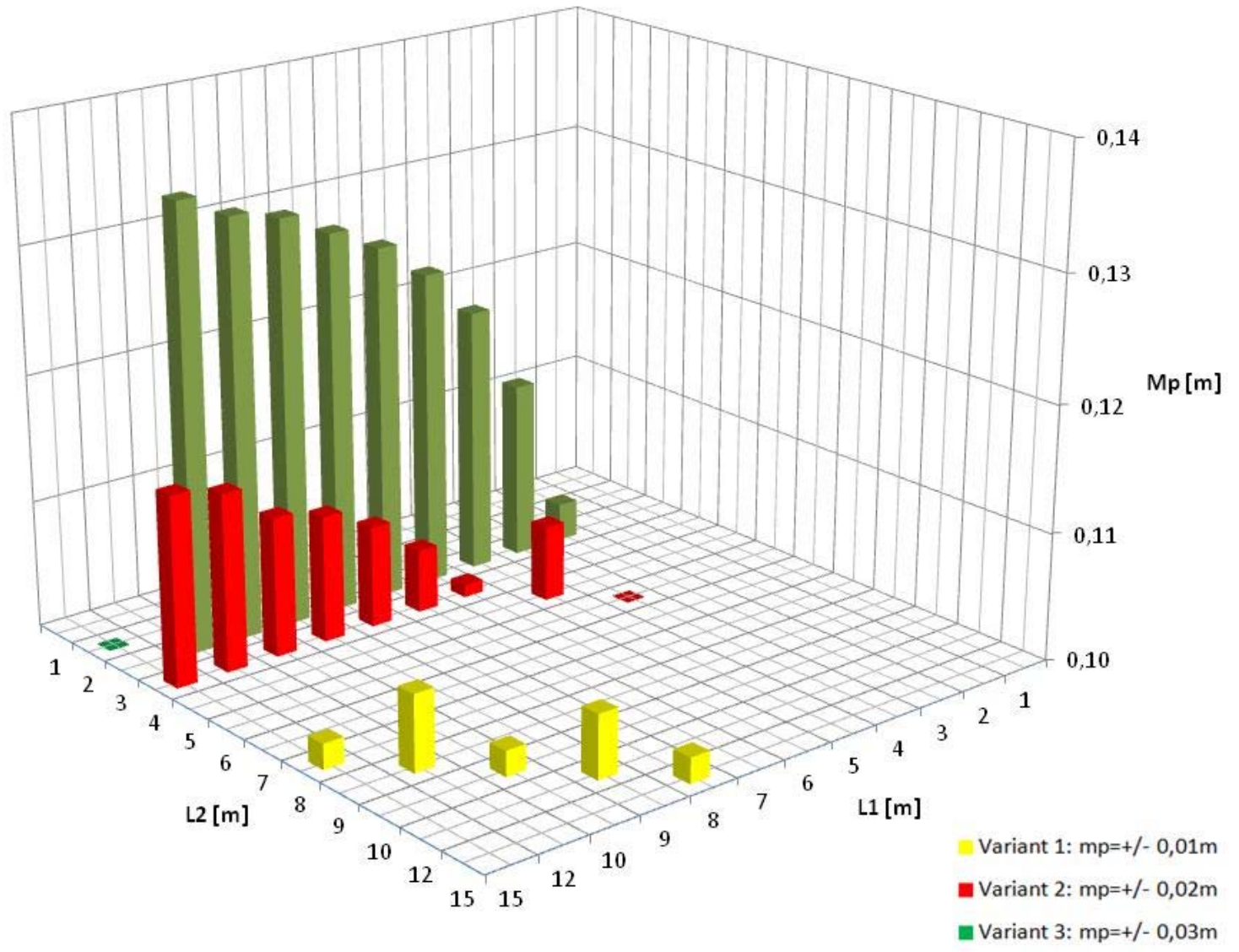

Fig. 2. Mean position error of the corner of the building $M_{P} \geq \pm 0.10 m$ for variants 1-3 
On the other hand, Tab. 2 and Fig. 2 demonstrate that the increase in the value of the mean position error of the corner of the building determined with reference to the control $\left(m_{P}= \pm 0.02 m\right)$ definitely restricts possibilities to combine the two measurement methods. In this case, the boundary value of the linear value (tie distance) for the wall face of the building is 3-4 meters. In this situation, although the criterion of (MIA, 2011), $\S 29$ section 1 is met, still a relatively large (nearly maximum) mean position error $M_{P}$ of the corner is obtained when determined in this measurement mode. Only linear measurements at the level of 1-2 meters ensure that the mean error $M_{P}$ of the determined corner point is of $\pm 0.04 \mathrm{~m}$ to $\pm 0.06 \mathrm{~m}$.

Variant 2 would find practical application in the field inn surveying the abovementioned vestibules, outbuildings, stairs, etc., while limiting their linear lengths to 2 meters.

The results demonstrated in Tab. 3 and Fig. 2 definitely restrict the use of the combination of the two surveying methods to a minimum. For variant 3 , the linear values of the wall faces of the building can not exceed $2 \mathrm{~m}$, with almost the maximum mean position error $M_{P}$ obtained. On the other hand, the value of the mean position error $M_{P}$ at the level of $\pm 0.06 \mathrm{~m}$ will be obtained only when the so-called tie distance does not exceed $1 \mathrm{~m}$. Thus, it can be stated that the practical application of the data from variant 3 only applies to the elements of buildings such as recesses or projections, as they usually reach the values of the tens of centimeters.

\section{Summary and conclusions}

The presented research results were to provide answer to the fundamental question: does the procedure of determining a position of some corners of a building, as often implemented by surveyors in practice, with reference to the other corner points of this building measured to the geodetic control meet the accuracy criteria, or should such procedure be completely eliminated? According to the regulation (MIA, 2011), § 29 section 1 , the basic accuracy criterion that must be met by each detail of the first-order accuracy is its mean position error $M_{P}$. This parameter was analyzed in detail, depending on the different assumptions which were adopted (3 variants). The conducted analysis demonstrated that the mean position error of output points, and therefore also of corners of a building, but determined with reference to the control, has the most significant influence on the formation of the parameter $M_{P}$ of the corner of a structure determined by the combination of the two surveying methods. Its value generates final research results and has a direct influence on permissible linear values (tie distances) of the surveyed walls of a building.

Before drawing the final conclusions, two issues need to be analyzed separately. First of all, according to the applicable regulations (MIA, 2011), § 29 section 1, such a procedure, which is often used by surveyors in practice, is not acceptable formally. It is substantiated by the fact that the position of a corner of a building is determined with reference to a corner of a building (a detail of the first-order accuracy with reference to the same type of a field detail). On the other hand, it is important to be aware that law should serve the society, not the other way round. In this context, having met the proposed criteria, it would be advisable to consider allowing for surveying certain structures in the analyzed mode. These criteria depend on the mean position error $m_{P}$ of corners of a building measured with reference to the control (from $\pm 0.01 \mathrm{~m}$ to \pm $0.03 \mathrm{~m}$ ) and the mean error of linear measurement $m_{l_{i}}= \pm 0.01 \mathrm{~m}$. For $m_{P}= \pm 0.01 \mathrm{~m}$, the lengths of the wall faces of the building can not exceed $7.00 \mathrm{~m}$. This is a rare case because it is unlikely that such a value of the error $m_{P}$ of the corner will be reached. 
On the other hand, for $m_{P}= \pm 0.02 m$, lengths of wall faces of a building can not exceed $4.00 \mathrm{~m}$. This situation is relatively common for vestibules, outbuildings, etc. It is far easier to meet this criterion for the parameter $m_{P}$, so in this case it would be possible to use a compilation of the subject surveying method. The last value of the error $m_{P}=$ $\pm 0.03 \mathrm{~m}$ restricts the use of this combination, making it applicable only to typical recesses or projections along wall faces of a building, which most frequently reach the values of the tens of centimeters. For this assumption, lengths of wall faces of a building can not exceed 1.00m.

In conclusion, it should be emphasized that the results of the conducted research studies demonstrate the possibility of applying a combination of the two surveying methods to determine a position of corners of a building, provided that the specified accuracy criteria are met.

\section{Acknowledgment}

This work was carried out within the statutory studies of the AGH University of Science and Technology, Faculty of Mining Surveying and Environmental Engineering, Department of Integrated Geodesy and Cartography No. 11.11.150.444

\section{References}

Andrianova, S. D. (2008). High-accuracy geodetic monitoring during construction and occupancy of modern buildings. Soil Mechanics and Foundation Engineering 2008. Volume 45, Issue 2, pp. 66-70. Doi:10.1007/s11204-008-9000-5

Buśko M. (2016). Analysis of the influence of changes in the laws of buildings in the aspect of updating the real etate cadastral database. Infrastructure and Ecology of Rural Areas. No. 2016/II/1 (Apr 2016)). pp. 395-410.

Buśko M. (2016). Building contour line in the database of the real estate cadastre in Poland pursuant to applicable laws. EconTechMod (Lublin): an international quarterly journal on economics in technology, new technologies and modeling processes. ISSN 2084-5715. - 2016 vol. 5 no. 3, pp. 183-190.

MIA. (2011). Regulation of the Minister of Internal Affairs and Administration of 9 November 2011 on the technical standards for the performance of geodetic topographic and detailed surveys as well as the preparation and transfer of these surveys to the National Cartographic Documentation Center database (in Polish). Journal of Laws 263 (item 1572), Warsaw: Government Legislation Center.

Ostwald M., Vaughan J. (2016). Measuring Architecture. The Fractal Dimension of Architecture. Book Section 2016. Springer International Publishing. Volume 1 of the series Mathematics and the Built Environment pp. 67-85. Doi: 10.1007/978-3-31932426-5_4

Przewięźlikowska A., Krzyżek R. (2016). As-built survey of buildings in the context of compliance of the location of the building with the site development plan for the plot). Geodesic Review. ISSN 0033-2127. 2016. 88 No. 8, pp. 11-15.

\section{Authors:}

Robert Krzyżek 1), rkrzyzek@agh.edu.pl

Anna Przewięźlikowska ${ }^{1)}$, przewie@agh.edu.pl

1) AGH University of Science and Technology,

Faculty of Mining Surveying and Environmental Engineering,

Department of Integrated Geodesy and Cartography

30 Mickiewicza Av., 30-059 Krakow, Poland 